\title{
GEOGRAFÍA
}

\section{Redes y estructura espacial, su papel en el desarrollo}

Recibido: 07/10/2016

Aprobado: 21/11/2016

\author{
Alicia Huamantinco Araujo \\ Universidad Nacional Mayor de San Marcos \\ < ahuamantincoa@unmsm.edu.pe >
}

\begin{abstract}
RESUMEN
La inserción del Perú en diferentes momentos de su historia, en la economía mundial como exportador de materia prima, se fue materializando en el territorio a través del establecimiento de las redes técnicas. Por su estrecha relación con las características físicas del territorio, las redes de circulación han tenido un papel relevante en la diferenciación de los espacios en el ámbito nacional, haciendo a unos lugares accesibles y articulados, y a otros marginales. Hoy en día, en que el modelo de modernización globalizadora, se sustenta en la tecnología y el conocimiento y en que los Estados y las regiones se preocupan por no quedar aislados, es imprescindible reflexionar sobre el reto que representa para el desarrollo regional el que el sistema vial del país - ferroviario y de carreteras - se haya construido no en función del desarrollo de la economía de las regiones, sino que fundamentalmente respondió al objetivo de articular las fuentes de materia prima con los mercados de destino, las regiones con los centros hegemónicos.
\end{abstract}

Palabras Clave: red vial, estructuras espaciales, modelo primario-exportador, desarrollo regional.

\section{Networks and spatial structure, their role in development}

\begin{abstract}
Peru's insertion, at different times in its history, in the world's economy as exporter of raw materials had been materializing in its territory through the establishment of the technical networks. Due to their close relationship with the physical characteristics of the territory, the circulation networks have played a key role in the differentiation of the spaces at the national level, making locations accessible and articulated or otherwise marginalized. Nowadays, when the global modernization model is based on technology and knowledge and the States and regions worry about being isolated, it is essential to reflect over the challenge that represents for regional development the fact that the country's transport system -railways and highways- was build not with the objetive to develop the economy of the regions but, fundamentally, to articulate the sources of raw material with the end markets, the regions with the hegemonic centers.
\end{abstract}

KEYwORDS: Transport network, spatial structures, primary-exporter model, regional development. 


\section{Introducción}

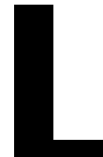
os sistemas de dominación históricamente establecidos entre países y regiones del mundo, han tenido como rasgo fundamental la explotación por una metropoli de los recursos de los colonias o de los países dominados económica y políticamente. Los recursos son apropiados por el centro a través de intercambios desiguales dentro del modelo centro-periferia. Los beneficios para las periferias son mínimos: infraestructura necesaria para esa explotación de recursos, establecimiento de servicios limitados especialmente en las ciudades, entre otros.

Las infraestructuras de transportes, en su evolución llegan a conformar redes y sistemas a nivel regional y nacional. En los tiempos actuales de revolución tecnológica, las redes, entre ellas las de transportes, alteran la relación espacio-tiempo y generan efectos estructurantes o desestructurantes en los territorios. Este tipo de transformaciones territoriales comienzan a aparecer en el Perú, en la década de 1990, con el auge de inversiones extranjeras constatándose que estas actividades privilegian determinadas regiones, mientras que otras quedan marginadas.

Entre las redes materiales o técnicas, una particular importancia tienen las redes de transportes. Como señala Bakis (1993) cuando hablamos del sistema de transportes es imposible ignorar su dependencia de superestructuras como: el contexto natural, nivel tecnológico, medio socioeconómico, estructura administrativa y legal, e inclusive la orientación política del poder.

En el caso del Perú, histórica y geográficamente, se constata la estrecha relación que la red de circulación (carreteras, caminos, vías férreas) con el territorio. En la región andina, a las redes de circulación les ha cabido un papel fundamental en el proceso de formación de la estructura espacial.

El presente artículo consta de tres partes. La primera comprende una revisión de conceptos, nociones sobre la producción de espacio y las estructuras espaciales y redes, entre ellas las de transportes. La segunda parte muestra una visión histórica de la conformación de la red vial nacional destacando el papel fundamental del modelo económico primario-exportador. La tercera, busca explicar desde la teoría geográfica de redes, la articulación de las redes con el desarrollo regional del país.

\section{Producción de espacio, estructura espacial y redes}

Consideramos necesario un rápido recorrido por la literatura científica, para precisar el objeto de estudio: las redes como parte de las estructuras espaciales, las mismas que son una expresión de cómo la sociedad ha organizado el espacio. En primer lugar ¡cómo entender el espacio geográfico?

\section{El espacio geográfico y las estructuras espaciales}

El espacio geográfico es producido por la sociedad, con sus acciones que se suceden en el tiempo. Los espacios se diferencian entre sí porque sociedades diferentes ejercen acciones diferentes y por consiguiente, producen lugares diferentes.

Henri Lefebvre, filósofo y geógrafo, en su libro Espacio y politica (1976) sostiene que el espacio desempeña un papel o una función decisiva en la estructuración de una totalidad, de una lógica, de un sistema, y que estaría esencialmente vinculado a la reproducción de las relaciones sociales de producción. La producción del espacio, para Lefébvre se encuentra en estrecha correspondencia con el nivel de las fuerzas productivas, ya que supone, por un lado, la utilización de las fuerzas y de las técnicas existentes; por otro, la iniciativa de grupos o clases capaces de intervenir y/o de concebir objetivos en determinada escala, actuando en un marco constitucional determinado, portadores de ideologías y principalmente de representaciones espaciales que corresponden a las relaciones de producción.

Milton Santos en su libro Espacio y método (1985) afirma que la sociedad solo puede ser definida a través del espacio, ya que el espacio es una consecuencia de su historia, más precisamente de la historia de los procesos productivos impuestos al espacio por la sociedad. Agrega, que cuando se estudia la organización espacial, los conceptos de forma, función, proceso y estructura son necesarios para explicar cómo el espacio social está estructurado, cómo los hombres organizan su sociedad en el espacio y cómo la concepción y el uso que el hombre hace del espacio experimentan cambios.

M. Santos en 1985 presentó una aproximación a la definición de estructura espacial:

... es algo así: una combinación localizada de una estructura demográfica específica, de una estructura de producción específica, de una estructura de renta 
específica, de una estructura de consumo específica, de una estructura de clases específica y de un arreglo específico de técnicas productivas y organizativas utilizadas por aquellas estructuras y que definen las relaciones entre los recursos presentes (Santos, 1985:17).

Las estructuras y los sistemas espaciales, no permanecen inertes, por el contrario están en proceso de cambio a través del tiempo. M. Santos, identifica tres principios en esta evolución:

1. el principio de la acción externa, responsable por la evolución exógena del sistema; 2. el intercambio entre subsistemas (o subestructuras), que permite hablar de una evolución interna del todo, una evolución endógena, y 3. la evolución particular de cada parte o elemento del sistema tomado separadamente, evolución que es igualmente interna y endógena (Santos, 1985:17).

Las redes en la geografia

Manuel Castells, define la red como una estructura espacial que presenta un patrón característico por donde se mueven flujos, es decir, una corriente o circulación de fluidos, personas o cosas que generan secuencias de intercambio e interacción determinadas y permiten conectar nodos o posiciones físicamente inconexas (Castells, M., 2000).

Milton Santos, citando a Curien, define las redes como:

Toda infraestructura que permite el transporte de materia, de energía o de información, y que se inscribe sobre un territorio donde se caracteriza por la topología de sus puntos de acceso terminales, sus arcos de transmisión, sus nudos de bifurcación o de comunicación (Curien, 1988:212) (Santos,1996:209),

subrayando que mediante las redes la apuesta no es la ocupación de las áreas, sino que la preocupación principal es la de activar puntos y líneas, o de crear otros.

Por su parte, Bakis (1993) sostiene que son redes tanto los meridianos y paralelos en la cartografía del globo como las redes hidrográficas, redes técnicas territoriales, redes de telecomunicaciones hertzianas, cuanto las redes urbanas. En el primer caso se trata de una proyección abstracta; en el segundo, de una proyección concreta de líneas de relaciones y ligaciones; y en el tercero, de la polarización de puntos de atracción y difusión.
A su vez, Machado (1993) señala que en la base de la noción de red está el reconocimiento de existencia de los «puntos» o nódulos, sin embargo no como puras abstracciones geométricas, ya que poseen una «espesura social y geográfica» (pueblos, ciudades, metrópolis, fábricas, centrales eléctricas, etc.). Representan nodosidades territoriales, entre las cuales los intercambios de materia, energía e información son necesariamente asimétricos y desiguales.

\section{Las redes de circulación y las redes de comunicación}

Las redes de circulación o de transportes, y las redes de comunicación son las dos fases de la movilidad. La circulación debe ser entendida como la transferencia de seres y de bienes, y la comunicación como la transferencia de información. Según Claude Raffestin (1993:200), la circulación y la comunicación "por ser complementarias, están presentes en todas las estrategias que los actores desencadenan para dominar las superficies y los puntos por medio de la gestión y del control de las distancias".

La circulación es visible por los flujos de hombres y de bienes que moviliza, por las infraestructuras que presupone y a través de las cuales el poder provee información sobre sí mismo. La red de circulación pone en realce la disposición de los territorios y las distancias. Toda la red de circulación, en el cuadro espaciotemporal, es por definición móvil y está en perpetua transformación dependiendo de la escala privilegiada por las estrategias y dependiendo de los intereses de los actores.

Así, la circulación se constituye en la imagen del poder según Raffestin, al afirmar que «un sistema de circulación es un instrumento creado, producido por actores, y es reproducido de una forma variable, en función de los proyectos políticos y económicos que evolucionan» (Raffestin, 1993:207).

Raffestin destaca que, la comunicación ha adquirido en la sociedad contemporánea una enorme importancia, relacionada con la ventaja que le proporciona al poder de "ver sin ser visto", permitiéndole controlar, interceptar, vigilar ocultamente. Raffestin (1993:203) afirma que «uno de los triunfos del poder es hoy informacional, y la informática es uno de los medios. El verdadero poder se desplaza para aquello que es invisible en gran parte, ya se trate de información política, económica, social o cultural». Los actores comprendieron así, que los medios cambian de lugar cada vez más en 
dirección a la información, cuya gestión y control son facilitados por las técnicas actuales y cuya naturaleza y utilización implica una determinada concepción del espacio y del tiempo.

En cada etapa del desarrollo de un espacio regional son necesarias las infraestructuras de circulación o transportes, aunque como es sabido, ellas por sí solas no garantizan el desarrollo y la integración de un determinado espacio. Los sistemas de infraestructura de transportes y comunicaciones, requieren de un conjunto de factores y condiciones para desempeñar un rol en el desarrollo. Las redes técnicas —que comprenden las redes de circulación y de comunicación- establecen potencialidades territoriales, relacionadas con la accesibilidad, conectividad y por las interrelaciones, por lo que tienen la capacidad de crear ventajas comparativas territoriales, que definen la competitividad espacial.

Este artículo se enfoca en el análisis de las redes de circulación, con el propósito de mostrar que las redes de circulación, constituyen un poderoso instrumento de estructuración del territorio.

Como destaca Raffestin (1993:205), citando a Chesnais, «la red de circulación permite concebir la naturaleza de la red geográfica, ya que esta red considera la disposición de los territorios y la distancia». A esto hay que sumar, que el estudio de la red de circulación permite una visión integral de la realidad. Al respecto, Bakis (1993) sostiene que se puede aplicar a todas las redes territoriales, lo que señala Richman para los transportes: que cuando hablamos del sistema de transportes es imposible ignorar su dependencia de las siguientes superestructuras: contexto natural, nivel tecnológico, medio socioeconómico, estructura administrativa y legal, e inclusive la orientación política del poder.

En el Perú, es relevante la estrecha relación que la red de circulación (carreteras, caminos, vías férreas), tiene con el territorio. En la región andina, caracterizada por las grandes altitudes y relieve accidentado, las redes de circulación han jugado un papel fundamental en el proceso histórico de formación de la estructura espacial. En el proceso de establecimiento de partes de estas redes, los lugares se fueron diferenciando en: accesibles-inaccesibles, próximos-distantes, incluidosexcluidos.

\section{Laconformación delared, vista desde un enfoque diacrónico}

Pensar en las redes en términos dinámicos, lleva a la intervención de las nociones de tiempo y espacio. San- tos (1996:209) destaca que «en sus relaciones con el territorio, las redes pueden ser examinadas según un enfoque genético y según un enfoque actual». A través del enfoque genético, las redes son vistas como un proceso en el cual partes de ellas se instalan en diversos momentos, diferentemente datados. La reconstitución de esa historia es compleja, pero es fundamental para entender la evolución de un lugar como una totalidad. Este estudio necesariamente es diacrónico.

El estudio de las redes desde el enfoque actual, según Santos:

Supone la descripción de lo que constituye un estudio estadístico de las cantidades y de las cualidades técnicas, pero también, la avaluación de las relaciones que los elementos de la red mantienen con la presente vida social, en todos sus aspectos, esto es, esa cualidad de servir como soporte corpóreo del cotidiano (Santos,1996:210) .

No sería posible aplicar de modo separado el enfoque genético y el enfoque actual, sobre todo porque, como afirma Santos (1996:210), "diacronía y sincronía, vistas a través del espacio geográfico son, exclusivamente, dos fases de un mismo fenómeno, o mejor aún, dos formas de percibir un movimiento unitario".

Raffestin (1993:209) apunta que "es conveniente, pues, descifrar las redes por medio de su historia y del territorio en el cual están instaladas, por medio de los modos de producción que han permitido su instalación y de las técnicas que les dieran forma».

En este artículo, se destacan momentos importantes en la configuración de la red vial nacional, en su relación con los procesos socioeconómicos y en su proyección hacia la actualidad

\section{La conformación de la red vial nacional, desde una visión histórica. El papel del modelo primario-exportador}

¿Cuáles son los antecedentes que explican la actual forma de organización del territorio? Consideramos que la clave de la respuesta está en los modelos económicos que se aplicaron en el Perú y en Latinoamérica. Al respecto, la geógrafa María Gudiño (2008) señala que el proceso de transnacionalización en América Latina se inició con su descubrimiento y "El resultado fue la constitución de dos modelos que prevalecieron hasta 
la actualidad. El modelo agroexportador en el que solo determinadas zonas geográficas, actividades y grupos sociales lograron integrarse al mundo al transformarse en productores de materias primas que demandaba el sistema internacional y el modelo tipo enclave en el que el objetivo fue la explotación de recursos naturales sin dejar nada a cambio sino simplemente mayor marginación. Es en estos antecedentes en donde se encuentran las causas que explican la actual forma de organización del territorio latinoamericano. Mientras algunas zonas concentran infraestructuras, equipamientos, actividades económicas y población, otras se encuentran sumidas en la pobreza y la marginación.”

El papel del crecimiento económico y urbano en los siglos XIX-XX en la conformación de la red vial

La actual trama vial del Perú tiene su origen en la segunda mitad del siglo XIX. El diseńo de la red vial es producto de un modelo impulsado desde el exterior, donde el puerto cumple un papel preponderante en el fortalecimiento del sistema, concentrando en la ciudad capital y en la Costa el crecimiento económico y dejando rezagadas y marginadas las otras regiones del país.

Según el Censo de 1876 la población del país era de 2699 mil habitantes, distribuida relativamente proporcional entre el norte, centro y sur del país. El patrón de asentamiento era disperso y predominaba la población rural. El mayor desarrollo urbano correspondía a los departamentos del litoral: Lima, Callao, Ica, Arequipa, Lambayeque, a los que se sumaba Junín. En los Andes, las «comunidades campesinas» predominaban en el sistema de propiedad; las haciendas concentraban menos de $30 \%$ de la población.

Un rol fundador en la trama vial del país, le correspondió a las vías férreas. Ellas fueron construidas en su mayor parte, entre mediados del siglo XIX y las primeras décadas del siglo $\mathrm{XX}$, desempeñando un papel fundamental en la conformación de las áreas de especialización como las de producción del azúcar y algodón, en el litoral; de lana y minerales en los Andes.

Al consolidarse esta especialización, se formaron enclaves económicos que condicionaron los patrones de asentamiento de la población en función a los nuevos centros de actividad económica regional. Se reforzó la estructura económica de exportación de materia prima: algodón, azúcar, petróleo, cobre y fierro, directamente ligados a la economía internacional, particularmente a los Estados Unidos. En el litoral, las haciendas de azúcar se transformaron en conglomerados. En los Andes del sur, la vía férrea que une Cusco, Puno y Arequipa con el puerto de Mollendo, puso las bases para la unidad regional del sur.

Un relevante papel sobre el crecimiento urbano, jugó la minería. Cuando la minería pasó a ser una actividad importante, la agricultura tradicional se retrajo progresivamente y se profundizó la situación de marginalidad de la población rural andina. La sistemática apropiación de las tierras de las comunidades aceleró la proletarización de los campesinos, que se incorporaron a la actividad minera. El proceso estuvo acompañado del crecimiento de los núcleos urbanos, así como de las concentraciones urbanas tipo enclave. La gran demanda de minerales, generada por la Primera Guerra Mundial, produjo el crecimiento urbano de los centros mineros. La vía férrea que une el puerto del Callao con las minas y ciudades de la sierra central, creó las bases de un espacio regional articulado.

Lima, la capital del país, en 1903 contaba con una población de 130 mil habitantes, desempeñaba el papel de centro del poder y de las actividades comerciales. Las ciudades que le seguían en la jerarquía urbana: Trujillo, Arequipa, Cajamarca y Ayacucho, tenían entonces entre 20 y 30 mil habitantes. Según Caravedo (1982), desde inicios del siglo XX, las demandas por alimentos en Lima y Callao no conseguían ser satisfechas por la producción de los valles vecinos. Así, estas ciudades pasaron a depender de las importaciones de alimentos del exterior.

El crecimiento urbano de Lima y de algunas otras ciudades, llevó a orientar la producción agropecuaria hacia ese mercado. Desde 1930, con la crisis internacional y la caída de las importaciones, se tomaron medidas que obligaban a las haciendas de los valles próximos a Lima a producir alimentos. En esa década, la ciudad de Arequipa, en el sur del país, se definió como el segundo centro urbano después de Lima.

\section{El modelo primario-exportador en el siglo XX y la consolidación del sistema centro-periferia nacional}

Hasta 1926, en el Perú las carreteras eran prácticamente inexistentes, con excepción de algunas pocas a nivel local. La circulación se hacía a través de una densa red de caminos. El mercado nacional estaba fragmentado en mercados regionales y locales, e incluso el comercio con el exterior tenía un dinamismo propio en cada región; sus oscilaciones repercutían 
más localmente que en el conjunto de la economía nacional. Los mercados regionales eran débiles a juzgar por la balanza comercial y por los movimientos de entrada y salida de mercancías por los puertos (Caballero, 1981). Los sectores urbanos de mayor desarrollo capitalista, exigían condiciones básicas para la circulación de las mercancías, formas más rápidas de articulación del territorio. Según Caravedo (1982), el problema nacional y la cuestión agraria se reducía a uno solo: el problema de la articulación vial. Los tiempos obligaban a cambios.

En el Perú, el período entre las dos guerras mundiales se caracterizó por el establecimiento y expansión del transporte por carretera y el declive de las vías férreas.

La diversidad de materias primas que el Perú exportaba, permitió un permanente flujo de divisas que facilitó el mantenimiento del modelo agro-minero exportador hasta la primera mitad del siglo XX. Las industrias que se instalaron a partir de 1920 eran nacionales y dedicadas a la manufactura con alto consumo de mano de obra. Entre 1920 y 1930 creció el número de empresas industriales y de comercio. En esta década se incrementa la importación de alimentos $(28 \%$ del total de las importaciones) y pasan a ser significativas las importaciones de maquinaria, herramientas y vehículos (Caravedo, 1982).

Después de la Primera Guerra Mundial se priorizó la construcción de la red vial nacional. La carretera Panamericana, considerada un producto de la doctrina Monroe de los Estados Unidos, fue construida entre 1920 y 1940, articulando el territorio a lo largo del litoral, articulándose con la carretera Panamericana del Ecuador y Chile.

La segunda postguerra significó una verdadera revolución por la construcción de nuevas vías de circulación y la incorporación del camión como medio de transporte. La carretera Panamericana se transformó en la columna vertebral de la red vial del país articulándose con vías orientadas a los puertos y valles litorales, y con vías transversales en dirección a los Andes y a la Amazonía. Entre las vías transversales, la más importante es la carretera Central, que articula los departamentos de Lima, Junín, Pasco y Huánuco. Su construcción fue iniciada en la década de 1930. Gracias a ello, áreas de economía tradicional del interior se articularon con las áreas modernizadas.

Con la construcción de la carretera Panamericana fue definida la configuración de la red vial del país, se fortaleció el dominio del litoral y se reforzó el carácter de la economía nacional orientada hacia el exterior.

Lo que señala Gudiño (2008) para el caso argentino, guarda similitud con lo que ocurre en el Perú: "El modelo agroexportador da lugar, en la primera mitad del siglo XX, a una etapa de sustitución de importaciones que acentúa el poder del nodo central". En el Perú, la década de 1940 marca el paso a la conformación de una economía que al lado de la producción de materia prima agropecuaria y minera, incorpora la producción industrial. De un lado, las demandas de los Estados Unidos durante la Segunda Guerra Mundial generaron el apoyo del Estado a las industrias orientadas a la exportación para ese país. Así, surge la metalmecánica y se expande la química y la pesca industrial. Por otro lado, desde inicios de la década de 1940, la producción industrial creció rápidamente orientada a la sustitución de importaciones.

La expansión de la industria motivada por el conflicto bélico, tuvo lugar en estrecha conexión con el capital mundial. Hasta 1940, se habían instalado 14 transnacionales en el país; a fines de la década ya eran 35 , varias de las cuales en el sector de alimentos. En julio de 1939, dos de las mayores transnacionales de producción de alimentos del mundo, se establecieron en el Perú para producir leche enlatada: la Nestlé, cuya sede central está en Suiza, y la General Milk Inc. (Carnation) con sede central en Los Angeles, Estados Unidos.

El primer censo del siglo XX, en 1940, presenta a Lima consolidada como la ciudad más importante del Perú, con una población de 601796 habitantes, lo que equivalía a $10 \%$ de la población total del país. La segunda ciudad, Arequipa, tenía 80 mil habitantes. En 1943, alrededor de 45\% de los establecimientos industriales estaban en Lima, y 17\% en el sur, especialmente en Arequipa (Caravedo, 1982). Para entonces las asignaciones presupuestales por parte del Estado, ya mostraba con claridad el privilegio de Lima y del litoral (Caravedo, 1982).

Las demandas de alimentos de la capital involucraban las zonas agrícolas de la sierra central, articulada con Lima por la carretera Central, así como las zonas al norte y sur de Lima, articuladas a través de la carretera Panamericana.

Las relaciones que se establecen entre el centro y la periferia nacional, nos remiten a la afirmación de Raffestin: 
La capital se apoya, en general, en una o en varias regiones, controla una o varias naciones o, en fin, se inscribe en grandes espacios. Porque ninguna capital puede poseer en sí misma los recursos necesarios para el ejercicio del poder. Ella puede reunir, drenar o colectar recursos útiles, pero es poco capaz de generarlos por sí misma (Raffestin, 1993:196).

\section{Relaciones de poder en la articulación de las redes con el desarrollo regional del país}

Las relaciones del poder constituyen una clave para la comprensión de las redes. Esto lo sostiene Raffestin (1993:157) quien define la red como el instrumento por excelencia del poder, destacando que las redes aseguran el control del espacio y que toda red es una imagen del poder o más exactamente, del poder de los actores dominantes. Respecto a la relación espacioredes-poder, Santos (1996:216) señala que «la propia estructura del espacio constituye una condición fundamental al ejercicio del poder y a la naturaleza local o regional de ese poder».

El modelo primario-exportador, preponderante a lo largo de la historia del país, está marcado por relaciones de inequidad en la relación centro-periferia. De acuerdo a Gonzales de Olarte (2000) la consolidación del modelo primario-exportador, ha definido las principales características del desarrollo regional peruano, que el autor identifica como las siguientes:

1) Concentración y centralización económica en la ciudad de Lima, consolidando un sistema centroperiferia y creando marginalidades muy profundas en la periferia.

2) Crecimiento divergente entre el centro y las periferias, entre las regiones más modernas y las más tradicionales, y entre el campo y la ciudad.

3) La persistencia de desigualdades productivas y distributivas materializadas territorialmente

La divergencia entre el modelo primario-exportador y las necesidades del desarrollo regional, la señala Epifanio Baca (1983) refiriéndose al papel de la red vial para el desarrollo del Cusco, al manifestar que se puede afirmar que las principales vías, de nivel nacional y departamental, que componen el sistema vial nacional, nunca fueron pensadas en función al desarrollo de la economía regional; ellas fueron construidas fundamentalmente con el objetivo de articular la región con los centros hegemónicos, como Lima y Arequipa, y para, a través de ellos, articularse con el exterior. Lo que el autor afirma en relación al Cusco, en general, continúa siendo válida para toda la región andina.

Esta divergencia se debe a que las redes de circulación, así como las de comunicación, son inseparables de los modos de producción. Por ello, su diseño, construcción y utilización depende de los medios a disposición: técnicos, sociopolíticos y socioeconómicos, así como de los objetivos de los actores.

La red vial del país fue evolucionando a través del proceso histórico, pasando del transporte por vía ferroviaria al transporte por carreteras. En concordancia con las etapas del modelo primario-exportador, cambiaron las fuentes de materia prima y las áreas de explotación. Por ello, M. Santos aporta a la comprensión del proceso cuando sostiene que necesitamos comprender la estructura social en cada período histórico para poder entender tanto la transformación de los elementos naturales en recursos sociales, cuanto el cambio que esos nuevos recursos (formas) sufren con el correr del tiempo. Seńala, que la sociedad establece los valores de diferentes objetos geográficos y los valores varían según la estructura socioeconómica específica de esa sociedad (Santos, 1985:54). Destacando que "en un caso como en otro el movimiento de cambio se debe a las modificaciones en los modos de producción concretos» (Santos, 1985:16).

Las mercancías, personas, información, energía - al tornarse flujos, presuponen la existencia de redes. Sostiene Bakis (1993) que la red contribuye para el funcionamiento de un sistema socioeconómico territorial, donde los flujos son respuestas a las necesidades de un momento preciso, que se manifiestan en el territorio. Tanto los flujos invisibles como los flujos de mercancías y de personas explican el funcionamiento de los conjuntos espaciales organizados. Ellos unen los elementos, asegurando relaciones internas (de los sistemas y subsistemas) y relaciones externas (entre los diferentes sistemas).

\section{El contradictorio potencial de las redes: solidarizar y excluir}

En un país andino como el Perú, las redes de circulación han jugado un papel fundamental en el proceso histórico de formación de la estructura espacial. Bajo la lógica del modelo primario-exportador partes de las redes fueron estableciéndose conectando los lugares donde se encontraban las fuentes de materia prima, con los 
lugares de consumo y con los puertos para la exportación. Como resultado, los lugares se fueron diferenciando en el tiempo, por su calidad como: accesiblesinaccesibles, próximos-distantes, incluidos-excluidos. Sobre este papel contradictorio, Dias (1995:148) sostiene que se debe a los organismos de gestión de la red, sea de gestión técnica, económica o jurídica, afirmando que "no son neutros, ellos colocan en juego relaciones sociales entre los elementos solidarizados con aquellos que permanecen marginalizados".

Las redes están presentes en las diversas escalas: a nivel mundial, nacional y local. Es a través de la conectividad, que la red solidariza los elementos. Pero, al mismo tiempo que tiene el potencial de conectar, de solidarizar, también tiene el de excluir. Por ello, Santos (1996:215) sostiene que "podemos reconocer, grosso modo, tres tipos o niveles de solidaridad, cuyo reverso son otros tantos niveles de contradicciones. Esos niveles son el nivel mundial, el nivel de los territorios de los Estados y el nivel local». En esta afirmación, el mundo aparece como primera totalidad, tornándose empírico por intermedio de las redes; la segunda totalidad es el territorio, país o Estado, que resulta de un contrato y cuyas fronteras están debilitándose debido a la globalización de las redes que comprometen el contrato; la tercera totalidad es el lugar. En el nivel local es "donde fragmentos de la red ganan una dimensión única y socialmente concreta, gracias a la ocurrencia, en la contigüidad, de fenómenos sociales agregados, basados en un acontecer solidario, que es el fruto de la diversidad y en un acontecer repetitivo, que no excluye la sorpresa» (Santos,1996:215).

\section{Referencias bibliográficas}

BACA, Epifanio (1983). Cusco: Sistemas viales, articulación y desarrollo regional. Cuadernos para el debate regional, Centro Bartolomé de las Casas. Cusco.
Bakis, Henry (1993). Les Réseaux et leurs Enjeux Sociaux. Presses Universitaires de France, Paris.

Bonilla, Heraclio (1982). «El Perú entre la Independencia y la Guerra con Chile». Historia del Perú. Tomo VI, ed. Mejía Baca, Barcelona, pp.395-473.

Caballero, José (1981). Economía agraria en la sierra peruana. Instituto de Estudios Peruanos, Lima.

Caravedo, Baltazar (1982). «Economía, producción y trabajo». Perú, siglo XX. Tomo VIII, ed. Mejía Baca, Barcelona. pp.191-361.

Castells, M. (2000). "La ciudad de la nueva economía", conferencia dictada en la Universidad de Barcelona, España. (http//:www.fbg.ub.es).

Cepes-Conveagro (2007). «TLC y agro preguntas pendientes». Equipo Técnico Cepes Conveagro 20.07.2007.

Dias, Leila (1995). «Redes: Emergência e Organização». In: Castro, Iná Elias de, Gomes, Paulo Cesar da Costa e CorrêA, Roberto Lobato (orgs.) Geografia: Conceitos e Temas, Bertrand Brasil, Rio de Janeiro, pp.141-162.

Gonzales de Olarte, Efraín (2000). Neocentralismo y neoliberalismo en el Perú. Instituto de Estudios Peruanos, Lima.

Gudiño, María (2008). Geografía de las Redes: Impacto en la Reconfiguración Escalar del Territorio Latinoamericano. Geo UERJ - Ano 10, v.2, n.18, $2^{\circ}$ semestre de 2008. P.1-23 www.e-publicacoes.uerj.br/index.php/geouerj/article/download/.../1178

Lefébvre, Henri (1976). Espacio y politica. El derecho a la ciudad, II. Ediciones Península, Barcelona.

Machado, Lia (1993). «A Geopolítica do Governo Local: Proposta de Abordagem aos Novos Territórios Urbanos da Amazônia». Anais do 3o Simpósio Nacional de Geografia Urbana UFRJ, Rio de Janeiro.

Raffestin, Claude (1993). Por uma Geografia do Poder. Ática, São Paulo.

Santos, Milton (1985). Espaço e Método. Nobel, São Paulo. Santos, Milton (1996). A Natureza do Espaço. Técnica e tempo, razão e emoção. HUCITEC, São Paulo. 